\title{
Efecto de un método de Entrenamiento Intervalado de Alta Intensidad sobre el consumo máximo de oxígeno en escolares chilenos
}

\author{
Effect of a High-intensity Interval Training method on maximum oxygen consumption in Chilean schoolchildren
}

\author{
Sergio Galdames-Maliqueo 1,5,6 orcid.org/0000-0002-0261-5741 \\ Álvaro Huerta-Ojeda ${ }^{2,3,5,6^{*}}$ orcid.org/0000-0001-6871-098X \\ Luis Chirosa-Ríos ${ }^{3}$ orcid.org/0000-0002-1008-176X \\ Pablo Cáceres-Serrano4 orcid.org/0000-0002-1691-9199 \\ Tomás Reyes-Amigo 1,5 orcid.org/0000-0001-6510-1230
}

1 Facultad de Ciencias de la Actividad Física y del Deporte, Universidad de Playa Ancha de Ciencias de la Educación. Valparaíso, Chile

2 Escuela de Educación Física, Facultad de Educación, Universidad de Las Américas sede Viña del Mar. Valparaíso, Chile

3 Grupo de Investigación y Desarrollo en Actividad Física, Salud y Deporte CTS 642, Facultad de Ciencias del Deporte, Universidad de Granada. Granada, España

4 Pontificia Universidad Católica de Valparaíso. Valparaíso, Chile

5 Grupo de Investigación en Salud, Actividad Física y Deporte ISAFYD, Universidad de Las Américas sede Viña del Mar. Valparaíso, Chile

6 Centro de Capacitación e Investigación Deportiva Alpha Sports. Valparaíso, Chile

Fecha de recepción: Mayo 17 - $2017 \quad$ Fecha de revisión: Septiembre 16-2017 Fecha de aceptación: Diciembre 1 - 2017

Galdames-Maliqueo S, Huerta-Ojeda A, Chirosa-Ríos L, Cáceres-Serrano P, Reyes-Amigo T. Efecto de un método de Entrenamiento Intervalado de Alta Intensidad sobre el consumo máximo de oxígeno en escolares chilenos. Univ. Salud. 2017;19(3):359-365. DOI: http://dx.doi.org/10.22267/rus.171903.98

\section{Resumen}

Introducción: Los bajos niveles de Consumo Máximo de Oxígeno ( $\mathrm{VO}_{2 \max }$ ) evaluados en escolares chilenos, sugieren la puesta en marcha de entrenamientos que mejoren la capacidad aeróbica. Objetivo: Analizar el efecto de un método de Entrenamiento Intervalado de Alta Intensidad sobre el Consumo Máximo de Oxígeno en escolares chilenos. Materiales y métodos: Treinta y dos escolares de octavo año básico divididos en dos grupos fueron parte del estudio (grupo experimental $=16$ y grupo control $=16$ ). La principal variable analizada fue el Consumo Máximo de Oxígeno a través del Test de Course Navette. Se aplicó un método de Entrenamiento Intervalado de Alta Intensidad basado en la Velocidad Aeróbica Máxima obtenida a través del Test. Para el análisis estadístico se utilizó un ANOVA Mixto. Resultados: El grupo experimental presentó un aumento significativo en el Consumo Máximo de Oxígeno entre el pre test y post test al ser comparado con el grupo control $(\mathrm{p}<0.0001)$. Conclusión: Los resultados del estudio mostraron un efecto positivo del Entrenamiento Intervalado de Alta Intensidad sobre el Consumo Máximo de Oxígeno. Al término del estudio, se concluye que el Entrenamiento Intervalado de Alta Intensidad es una buena metodología de estimulación para escolares chilenos.

Palabras clave: Consumo de oxígeno; adaptación fisiológica; niño. (Fuente: DeCS, Bireme).

\begin{abstract}
Introduction: The low levels of maximum oxygen consumption (VO2max) evaluated in Chilean schoolchildren suggest the startup of trainings that improve the aerobic capacity. Objective: To analyze the effect of a Highintensity Interval Training method on maximum oxygen consumption in Chilean schoolchildren. Materials and methods: Thirty-two high school students from the eighth grade, who were divided into two groups, were part of the study (experimental group = 16 students and control group = 16 students). The main analyzed variable was the
\end{abstract}


maximum oxygen consumption through the Course Navette Test. A High-intensity Interval training method was applied based on the maximum aerobic speed obtained through the Test. A mixed ANOVA was used for statistical analysis. Results: The experimental group showed a significant increase in the Maximum Oxygen Consumption between the pretest and posttest when compared with the control group $(p<0.0001)$. Conclusion: The results of the study showed a positive effect of the High-intensity Interval Training on the maximum consumption of oxygen. At the end of the study, it is concluded that High-intensity Interval Training is a good stimulation methodology for Chilean schoolchildren.

Keywords: Oxygen consumption; adaptation, physiological; child. (Source: DeCS, Bireme).

\section{Introducción}

Chile se vio sorprendido por los malos resultados obtenidos en el Estudio Nacional de Educación Física del año 2014. En dicha evaluación, además de evidenciarse resultados "no satisfactorios" en las pruebas de salto a pies juntos, abdominales cortos, flexión de tronco adelante y Test de Course Navette ( $\mathrm{t}-\mathrm{CN}$ ), quedaron expuestos los altos índices de sobrepeso y obesidad en estudiantes de edad puberal. Esta medición reveló que el $68 \%$ de la población escolar (mujeres y hombres) presentan una condición "necesita mejorar" en su consumo máximo de oxígeno $\left(\mathrm{VO}_{2 \max }\right)^{(1)}$.

El $\mathrm{VO}_{2 \max }$ es considerado como un marcador de la condición física y la buena salud, independiente de la edad y el género(2). En un estudio publicado el 2012, Mayorga-Vega et al.(3), concluyeron que los niños y niñas de 10-12 años sin sobrepeso presentan mayores valores de $\mathrm{VO}_{2 \max }$ que sus pares con sobrepeso u obesidad. Es un hecho que el $\mathrm{VO}_{2 \text { max }}$ refleja el funcionamiento del sistema cardiorrespiratorio, siendo este sistema uno de los componentes más estudiado por los profesionales de las ciencias del ejercicio en las últimas dos décadas(4). Tanto es así, que Ramírez-Lechuga et al.(5), debido a los bajos niveles de $\mathrm{VO}_{2 \max }$ evaluados, sugirieron de forma urgente la puesta en marcha de programas de entrenamiento que mejoren la capacidad aeróbica de los adolescentes; más aún, con el fin de obtener mejores estándares de condición física, y con esto mejor salud y calidad de vida.

En el año 2010 la Organización Mundial de la Salud cambió la recomendación de actividad física en poblaciones de 5 a 17 años, sugirió una actividad física diaria de al menos 60 minutos con una intensidad de moderada a vigorosa(6).
Varios estudios coinciden que la resistencia aeróbica es un indicador de $\operatorname{salud(1-3,7,8)}$ y que altos Índices de Masa Corporal (IMC), junto a elevados porcentajes de grasa corporal se relacionan inversamente con la capacidad cardiorrespiratoria en escolares ${ }^{(9)}$. Sumado a lo anterior, se ha evidenciado que los mayores cambios funcionales que pueden favorecer al mejoramiento de la capacidad, se deben realizar en fase sensible de la resistencia (entre los $11 \mathrm{y}$ los 15 años), ya que es en esta etapa donde se ve una mayor efectividad en la capacidad de entrenamiento aeróbico(10).

La Velocidad Aeróbica Máxima (VAM), es un parámetro de intensidad que permite desarrollar la potencia aeróbica máxima. La VAM es un parámetro fisiológico importante, ya que implica conocer la velocidad porcentual de carrera a que está siendo sometido un sujeto(11). Actualmente, se han aplicado métodos de entrenamiento utilizando estos parámetros ${ }^{(12,13)}$. Una prueba de campo indirecta que permite obtener la VAM es el t-CN4, si bien, el t-CN ha sido aplicado por Agencia de Calidad de la Educación para estimar el $\mathrm{VO}_{2 \max }$ en escolares chilenos(1), las cargas de trabajo basadas en la VAM obtenida a través de esta prueba de campo no han sido del todo empleadas en escolares. Por lo tanto, se desconoce si es posible mejorar el $\mathrm{VO}_{2 \max }$ en poblaciones escolares de octavo año básico, aplicando un método de Entrenamiento Intervalado de Alta Intensidad, (HIIT por sigla en inglés) basado en la VAM obtenida a través del tCN adicional a la clase de Educación Física.

El objetivo principal del estudio fue analizar el efecto de un método HIIT basado en la VAM obtenida a través del $\mathrm{t}$-CN sobre el $\mathrm{VO}_{2 \max }$ en escolares chilenos. Como objetivo secundario, se 
evaluó el tiempo máximo realizado en el t-CN por los escolares.

\section{Materiales y métodos}

Este estudio es de tipo cuasi experimental con pre test y post test, mientras que el protocolo aplicado fue un HIIT basado en la $\operatorname{VAM}^{(4)}$ obtenida a través del t-CN.

La muestra estuvo constituida por 32 adolescentes varones de octavo año básico del sistema escolar chileno. Esta muestra fue escogida de manera no probabilística. Una vez realizado el pre test, los sujetos fueron ordenados por rendimiento y asignados tanto al grupo experimental (GE) (16 casos) como al grupo control (GC) (16 casos) por conveniencia (impares al grupo experimental y pares al grupo control) (Tabla 1). Antes de comenzar la aplicación del tratamiento, tanto los padres como los adolescentes firmaron un consentimiento y asentimiento informado respectivamente.

Tabla 1. Características de la muestra (media \pm DS)

\begin{tabular}{lcr}
\hline & $\begin{array}{c}\text { Grupo experimental } \\
\text { (n= 16) }\end{array}$ & $\begin{array}{c}\text { Grupo control } \\
(\mathbf{n = 1 6 )}\end{array}$ \\
\hline Edad (años) & $13,1 \pm 0,3$ & $13,4 \pm 0,5$ \\
Estatura (cm) & $166,6 \pm 7,1$ & $161,9 \pm 4,3$ \\
Peso (Kg) & $56,5 \pm 7,9$ & $56,0 \pm 6,9$ \\
IMC (Kg/m $\left.{ }^{2}\right)$ & $20,3 \pm 2,6$ & $21,3 \pm 2,6$ \\
\hline IMC (índice de masa corporal); DS (desviación standard) &
\end{tabular}

\section{Procedimientos}

El peso y la estatura se midieron con la balanza y estadiómetro Health o Meter Professional ${ }^{\circledR}$, mientras que el control del tiempo máximo realizado en el t-CN se midió con un cronómetro manual Casio HS-70W囚. Para evaluar el $\mathrm{VO}_{2 \max }$ antes y después de aplicado el HIIT, se aplicó el t$\mathrm{CN}$. Posteriormente, para cada sujeto se obtuvo la $\mathrm{VAM}^{4}$ y el $\mathrm{VO}_{2 \max }{ }^{(14)}$ a través del $\mathrm{t}-\mathrm{CN}$. Sin embargo, y pese a que en este estudio no se realizó una cuantificación de horas entrenamiento físico extras para ninguno de los grupos, se sugiere aplicar un cuestionario para el recuento de estas actividades durante el desarrollo de la intervención.
El HIIT aplicado tuvo una duración de 16 sesiones. Para la intervención con el GE, la aplicación del HIIT se realizó de la siguiente manera: se confeccionó un recorrido con un largo de $20 \mathrm{~m}$. El recorrido consistió en desplazamientos lineales con cambios de dirección de 180 grados terminado los $20 \mathrm{~m}$. En relación al número de cambios de dirección, estos iban en relación a la distancia que debía recorrer cada sujeto en los $10 \mathrm{~s}$ de estímulo. Por ejemplo: si un sujeto en $10 \mathrm{~s}$ debía recorrer $36 \mathrm{~m}$, sólo tenía un cambio de dirección, pero si debía recorrer $44 \mathrm{~m}$, debía realizar dos cambios de dirección (a los $20 \mathrm{~m}$ y a los $40 \mathrm{~m}$ ). Una vez realizada la distancia por repetición en los $10 \mathrm{~s}$, los sujetos caminaban hasta el punto de salida. Así completaban los $20 \mathrm{~s}$ de pausa, para luego realizar la siguiente repetición.

Las cargas aplicadas en el tratamiento experimental fueron individualizadas para cada participante del GE. Además, durante el desarrollo del protocolo hubo progresiones metodológicas relacionadas con la carga. Para determinar los protocolos y fórmulas para la velocidad aeróbica máxima y la estimación del consumo máximo de oxígeno en base al t-CN, se utilizaron las ecuaciones de García et al.(4) y de Ruiz et al.(14) respectivamente. El detalle del tratamiento experimental, y las progresiones de las cargas están reportadas en la Tabla 2 y en la Figura 1.

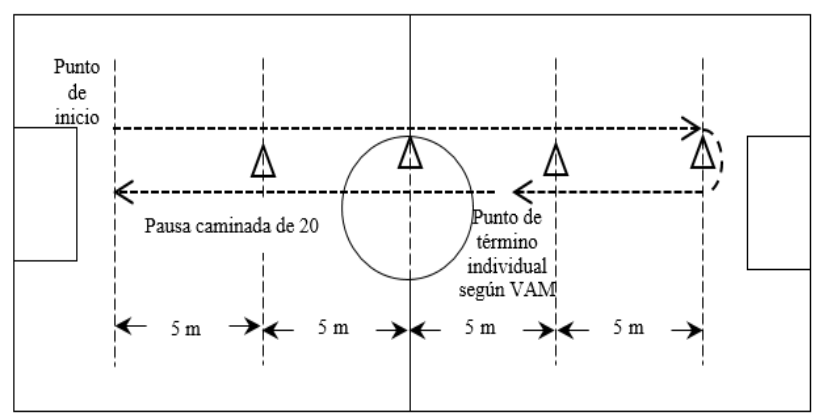

( $\Delta$ (conos);--------(dirección del recorrido); VAM (velocidad aeróbica máxima).

Figura 1. Distribución de materiales y secuencia de recorrido de las series 
Tabla 2. Estructura del programa de Entrenamiento Intervalado de Alta Intensidad con base a la velocidad aeróbica máxima del Test de Course Navette

\begin{tabular}{|c|c|c|c|c|c|c|c|c|}
\hline & $\begin{array}{l}\text { Microciclo } 1 \\
(2 \text { sesiones) }\end{array}$ & $\begin{array}{l}\text { Microciclo } 2 \\
\text { (2 sesiones) }\end{array}$ & $\begin{array}{l}\text { Microciclo } 3 \\
\text { (2 sesiones) } \\
\end{array}$ & $\begin{array}{l}\text { Microciclo } 4 \\
\text { (2 sesiones) }\end{array}$ & $\begin{array}{l}\text { Microciclo } 5 \\
\text { (2 sesiones) }\end{array}$ & $\begin{array}{l}\text { Microciclo } 6 \\
\text { (2 sesiones) } \\
\end{array}$ & $\begin{array}{l}\text { Microciclo } 7 \\
\text { (2 sesiones) }\end{array}$ & $\begin{array}{l}\text { Microciclo } 8 \\
\text { (2 sesiones) }\end{array}$ \\
\hline Intensidad (VAM) & $100 \%$ & $100 \%$ & $105 \%$ & $100 \%$ & $105 \%$ & $105 \%$ & $110 \%$ & $105 \%$ \\
\hline Series & 3 & 3 & 3 & 3 & 3 & 3 & 3 & 3 \\
\hline Repeticiones & 10 & 12 & 14 & 11 & 12 & 13 & 14 & 11 \\
\hline Tiempo repetición (s) & 10 & 10 & 10 & 10 & 10 & 10 & 10 & 10 \\
\hline Pausa (s) & 20 & 20 & 20 & 20 & 20 & 20 & 20 & 20 \\
\hline Macropausa (min) & 2 & 2 & 2 & 2 & 2 & 2 & 2 & 2 \\
\hline Tiempo efectivo (min) & $5 \mathrm{~min}$ & $6 \mathrm{~min}$ & $7 \mathrm{~min}$ & $5 \min 30 s$ & $6 \mathrm{~min}$ & $6 \min 30 s$ & $7 \mathrm{~min}$ & $5 \min 30 s$ \\
\hline Tiempo total & $19 \mathrm{~min}$ & $22 \mathrm{~min}$ & $25 \mathrm{~min}$ & $20.5 \mathrm{~min}$ & $22 \mathrm{~min}$ & $23.5 \mathrm{~min}$ & $25 \mathrm{~min}$ & $20.5 \mathrm{~min}$ \\
\hline Volumen (m) & 1230 & 1476 & 1808 & 1353 & 1550 & 1679 & 1894 & 1421 \\
\hline
\end{tabular}

\section{Análisis estadístico}

Los datos se analizaron de la siguiente manera y orden: las variables de $\mathrm{VO}_{2 \max }$ y tiempo máximo en el $\mathrm{t}-\mathrm{CN}$, fueron sometidos a la prueba de normalidad Kolmogorov-Smirnov (K-S). Para comparar el comportamiento de las variables durante la aplicación del HIIT, se aplicó un ANOVA mixto. El tamaño del efecto (TE) para este análisis se calculó utilizando la prueba Eta Cuadrado Generalizado. Para el análisis Post hoc de ambas variables se utilizó la prueba $t$ de Student. Él TE para este análisis se calculó utilizando la prueba $d$ de Cohen. El análisis de datos se realizó con el software Graphpad Instat Versión $3.05^{\circledR}$.

\section{Consideraciones éticas}

El estudio es considerado como de riesgo mínimo de acuerdo a la Declaración de Helsinki. El estudio junto con el consentimiento y asentimiento informado fueron aprobados por el Comité de Investigación Humana de la Universidad de Granada, España.

\section{Resultados}

Tabla 3. $\mathrm{VO}_{2 \max } \mathrm{y}$ tiempos obtenidos en el t-CN para el GE y GC en pre test y post test

\begin{tabular}{|c|c|c|c|c|c|c|}
\hline \multicolumn{7}{|c|}{ Análisis del $\mathrm{VO}_{2 \max }$ a través de un ANOVA mixto } \\
\hline & \multicolumn{2}{|c|}{ GE } & \multicolumn{2}{|c|}{ GC } & \multirow[b]{2}{*}{$\begin{array}{l}\text { ANOVA } \\
\text { Mixto }\end{array}$} & \multirow[b]{2}{*}{$\begin{array}{l}\text { Eta Cuadrado } \\
\text { Generalizado }\end{array}$} \\
\hline & $\begin{array}{c}\text { Pre test } \\
\text { media } \pm \text { DS }\end{array}$ & $\begin{array}{c}\text { Post test } \\
\text { media } \pm \text { DS }\end{array}$ & $\begin{array}{c}\text { Pre test } \\
\text { media } \pm \text { DS }\end{array}$ & $\begin{array}{c}\text { Post test } \\
\text { media } \pm \text { DS }\end{array}$ & & \\
\hline $\mathrm{VO}_{2 \max } \cdot\left(\mathrm{ml} \cdot \mathrm{Kg}^{-1} \cdot \mathrm{min}^{-1}\right)$ & $39,65 \pm 5,96$ & $41,95 \pm 6,17$ & $40,09 \pm 6,33$ & $39,59 \pm 6,83$ & $* * *$ & 0,01 \\
\hline \multicolumn{7}{|c|}{ Análisis del tiempo máximo en el t-CN a través de un ANOVA mixto } \\
\hline & \multicolumn{2}{|c|}{ GE } & \multicolumn{2}{|c|}{ GC } & & \\
\hline & $\begin{array}{c}\text { Pre test } \\
\text { media } \pm \text { DS }\end{array}$ & $\begin{array}{c}\text { Post test } \\
\text { media } \pm \text { DS }\end{array}$ & $\begin{array}{c}\text { Pre test } \\
\text { media } \pm \text { DS }\end{array}$ & $\begin{array}{c}\text { Post test } \\
\text { media } \pm \text { DS }\end{array}$ & ANOVA Mixta & $\begin{array}{l}\text { Eta Cuadrado } \\
\text { Generalizado }\end{array}$ \\
\hline Tiempo (s) & $299,1 \pm 129,6$ & $350,0 \pm 141,9$ & $313,3 \pm 140,4$ & $301,5 \pm 156,6$ & $* * *$ & 0,01 \\
\hline
\end{tabular}


Aplicado un ANOVA Mixto, se evidenció una diferencia significativa en el $\mathrm{VO}_{2 \max }$ para el GE ( $\mathrm{p}$ $<0,0001 ; \mathrm{TE}=0,01$ ). En el análisis Post hoc, una vez aplicada la prueba $t$, sólo el GE evidenció cambios significativos en el $\mathrm{VO}_{2 \max }$ entre el pre test y post test (GE: $\mathrm{p}=0,0001, \mathrm{TE}=0,379$; Grupo Control (GC): p < 0,33, TE = 0,076). En relación al tiempo máximo realizado en el t-CN, el ANOVA Mixto mostró incrementos significativos en el GE entre el pre test y post test al ser comparado con el GC ( $p<0,0001$, TE $=$ 0,01). En el análisis Post hoc sólo el GE presentó incrementos significativos (GE: $p<0,0001, \mathrm{TE}=$ $0,080 ; \quad \mathrm{GC}: \mathrm{p}=0,31, \mathrm{TE}=0,375)$. Las progresiones están reportadas en la Tabla 3.

\section{Discusión}

En relación al objetivo principal de la investigación, al término del Entrenamiento Intervalado de Alta Intensidad, el GE evidenció aumentos significativos en el consumo máximo de oxígeno estimado a través del $\mathrm{t}-\mathrm{CN}$. De manera paralela, el tiempo máximo de ejecución en t-CN mostró un incremento significativo al término de la intervención en el GE.

El HIIT empleado en la intervención del presente estudio, se basó en la VAM individual obtenida desde el $\mathrm{t}-\mathrm{CN}^{(4)}$. Al igual que el presente estudio, existen otras investigaciones con protocolos con base a pruebas de campo(15,16). Dentro de éstas, Marta et al.(16), estudiaron las variaciones de $\mathrm{VO}_{2 \max }$ posterior a un entrenamiento con el $75 \%$ de la VAM obtenida a través del t-CN. Es importante mencionar que estos investigadores usaron un método continuo y no un HIIT, por lo tanto, los resultados evidenciados en ese estudio son difíciles de comparar con los de esta investigación, más aún, los autores relacionaron los Estadios de Tanner (maduración biológica) con la influencia en la capacidad de entrenamiento de los sujetos, evidenciando que en los estadios I y II los sujetos mostraron cambios significativos en el $\mathrm{VO}_{2 \max }(\mathrm{p}<0.01)$. En estudios similares, pero con adolescentes obesos con insulino resistencia, se reportó una reducción en el Índice de Masa Corporal y perímetro de cintura-cadera luego de dos meses de entrenamiento de resistencia $(p<0,001)^{(17)}$; desafortunadamente, en este estudio no se utilizó una VAM proveniente del t-CN para programar las cargas de entrenamiento ni el HIIT como método para desarrollar el $\mathrm{VO}_{2 \text { max }}$.

Córdova et al.(18), investigaron la relación entre el $\mathrm{VO}_{2 \max } \mathrm{y}$ los niveles de actividad física. Al término del estudio los investigadores comprobaron que los adolescentes insertos en deportes presentaron un $\mathrm{VO}_{2 \max }$ significativamente más alto que sus pares sedentarios y activos ( $\mathrm{p}<$ $0,001)$. Estos autores expusieron que un valor más elevado de $\mathrm{VO}_{2 \max }$ en edades puberales estaría directamente relacionado con una mejor condición física en edades adultas, reduciendo así los factores de riesgo cardiovascular(18). Sumado a lo anterior, Tomkinson et al.(19), concluyeron que las causas de la disminución del rendimiento en las personas está ligado a la disminución de la actividad física en la comunidad, responsabilizando al avance de tecnologías que promueven el sedentarismo, además de la fácil disponibilidad de alimentos altos en calorías. Esto permite afirmar que un aumento del $\mathrm{VO}_{2 \max }$ genera una mejora en el sistema cardiorrespiratorio, incrementando las capacidades aeróbicas de los alumnos y de forma directa la salud de la población.

Como se mencionó en párrafos anteriores, esta investigación se basó en un HIIT estructurado en la VAM obtenida del t-CN(4). En relación a esto, planificar las sesiones de entrenamiento de forma individualizada, controlando variables como la intensidad, el volumen o la pausa con base a pruebas de campo, es fundamental para conseguir adaptaciones orgánicas y de condición física(20,21), específicamente un incremento en el consumo máximo de oxígeno en escolares(22). En un estudio realizado por Berthoin et al(23), se determinó el efecto de dos programas de entrenamiento en base a la VAM obtenida del test de la Universidad de Montreal en estudiantes entre 14 a 17 años; el primer programa definido como entrenamiento moderado ocupó el 85\% de la VAM, mientras que el segundo definido como entrenamiento intenso ocupó entre el 90 y el $120 \%$ de la VAM; los investigadores concluyeron que sólo el entrenamiento intenso generó cambios 
significativos en la VAM de hombres y mujeres ( $\mathrm{p}$ $<0,001)$. Si bien el estudio de Berthoin et al(23) es concluyente, estos investigadores no utilizaron el t-CN como parámetro de control y programación de cargas de entrenamiento, mientras que para este estudio fue fundamental el uso del t-CN, ya que este test es utilizado por la Agencia de Calidad de Educación para evaluar la condición física en escolares chilenos(1).

Junto al estudio presentado por Marta et al(16), existen otros que evaluaron y/o programaron las sesiones de entrenamiento basados en el t-CN. Así quedó demostrado en un estudio realizado por Reyes(24), quien evaluó el $\mathrm{VO}_{2 \max }$ antes y después de la aplicación de un programa de actividad física. Al término del estudio, el autor reportó cambios significativos en el $\mathrm{VO}_{2 \max }(\mathrm{p}<$ $0,05)$. Sin embargo, a diferencia de ese estudio, esta investigación usó muestras con un $\mathrm{VO}_{2 \max }$ igual antes de la intervención, mientras que el programa quedó totalmente definido (Figura 1) (Tabla 2). Por lo anterior, cualquier tipo de modificación tanto en el $\mathrm{VO}_{2 \max }$ como en el tiempo máximo realizado en el $\mathrm{t}-\mathrm{CN}$, fue atribuido a la aplicación del HIIT.

\section{Conclusión}

Al término de la aplicación del HIIT, el GE evidenció aumentos significativos en el $\mathrm{VO}_{2 \max }$. De igual manera, el tiempo máximo de ejecución en t-CN en el GE, mostró un incremento significativo al término de la intervención.

\section{Limitaciones}

Dadas las condiciones específicas de la población y el tamaño de la muestra, es aventurado hacer inferencias y extrapolaciones de los resultados para grupos etarios con similares características. Sin embargo, tanto los profesores de Educación Física como alumnos pudieron observar una mejor aplicabilidad del t-CN, ya que de esta prueba se desprendieron los programas individualizados de entrenamiento.

Conflicto de interés: Los autores declaran no tener conflicto de intereses.

\section{Referencias}

1. Chile. Agencia de Calidad de la Educación. Informe de Resultados Estudio Nacional Educación Física 2014. Santiago de Chile: Agencia de Calidad de la Educación; 2015. Disponible en: http://archivos.agenciaeducacion.cl/Estudio_Nacional_ Educacion_Fisica_2014_8basico.pdf

2. Bassett D, Howley E. Limiting factors for maximum oxygen uptake and determinants of endurance performance. Med Sci Sports Exerc. 2000;32(1):70-84.

3. Mayorga-Vega D, Podadera A, Tejero M, Marban R. Asociación del IMC y el nivel de condición física en escolares de educación primaria. J Sport Health Res. 2012;4(3):299-310.

4. García G, Secchi J. Relationship between the final speeds reached in the 20 metre course navette and the MAS-EVAL test. A proposal to predict the maximal aerobic speed. Apunts Med Esport (English Edition). 2013;48(177):27-34.

5. Ramírez-Lechuga J, Femia P, Sánchez-Muñoz C, Zabala M. La actividad física en adolescentes no muestra relación con el consumo máximo de oxígeno. Arch Med Deporte. 2011;28(142):103-12.

6. Organización Mundial de la Salud. Recomendaciones mundiales sobre actividad física para la salud. Ginebra: OMS; $2010 . \quad$ Disponible en: http://apps.who.int/iris/bitstream/10665/44441/1/9 789243599977_spa.pdf Consultado [16/09/2017].

7. Muros J, Morente-Sánchez J, Díaz M. Efecto de un programa de actividad física sobre el rendimiento aeróbico y la fuerza de prensión manual en niños. Arch Med Deporte. 2014;31(1):9-13.

8. Gálvez A, Rodríguez P, García-Cantó E, Rosa A, PérezSoto J, Tarraga L, et al. Capacidad aeróbica y calidad de vida en escolares de 8 a 12 años. Clin Investig Arterioscler. 2015;27(5):239-45.

9. López-Jaramillo $\mathrm{P}$, Gómez-Arbeláez D, Cohen D, Camacho P, Rincón-Romero K, Hormiga $C$, et al. Asociación entre obesidad y baja capacidad muscular y función cardiorrespiratoria, factores de riesgo cardiometabólico en niños colombianos. Trauma Fund MAPFRE. 2013;24(1):17-23.

10. Winter R. Le fasi sensibili (parte prima). Rivista di Cultura Sportiva. V. 1986;6:8-10.

11. García G, Secchib J, Arcuric C. Relación entre las velocidades finales alcanzadas en los test UMTT y UNCa en sujetos masculinos. Apunts Med Esport. 2016;51(190):48-54.

12. Casas A. Physiology and methodology of intermittent resistance training for acyclic sports. J Hum Sport Exerc. 2008;3(1):23-52.

13. Ramírez-Lechuga J, Muros-Molina J, Morente-Sánchez J, Sánchez-Muñoz C, Femia- Marzo P, Zabala-Díaz M. Efecto de un programa de entrenamiento aeróbico de 8 semanas durante las clases de educación física en adolescentes. Nutr Hosp. 2012;27(3):747-54.

14. Ruiz J, Ramírez-Lechuga J, Ortega F, Castro-Piñero J, Benítez J, Arauzo-Azofra A, et al. Artificial neural network-based equation for estimating V02max from 
the $20 \mathrm{~m}$ shuttle run test in adolescents. Artif Intell Med. 2008;44(3):233-45.

15. García C, Secchi J, Cappa D. Comparación del consumo máximo de oxígeno predictivo utilizando diferentes test de campo incrementales: UMTT, VAM-EVAL y $20 \mathrm{~m}-$ SRT. Arch Med Deporte. 2013;30(3):156-62.

16. Marta $C$, Marinho $D$, Izquierdo $M$, Marques $M$. Differentiating maturational influence on training-induced strength and endurance adaptations in prepubescent children. Am J Hum Biol. 2014;26(4):469-75.

17. Ounis O, Elloumi M, Chiekh I, Zbidi A, Amri M, Lac G, et al. Effects of two-month physical-endurance and dietrestriction programmes on lipid profiles and insulin resistance in obese adolescent boys. Diabetes Metab. 2008;34(6):595-600.

18. Córdova A, Villa G, Sureda A, Rodríguez-Marroyo J, Sánchez-Collado M. Actividad física y factores de riesgo cardiovascular de niños españoles de 11-13 años. Rev Esp Cardiol. 2012;65(7):620-6.

19. Tomkinson G, Léger L, Olds T, Cazorla G. Secular trends in the performance of children and adolescents (19802000). Sports Med. 2003;33(4):285-300.

20. Nicola B. La incidencia fisiológica de los parámetros de duración, intensidad y recuperación en el ámbito del entrenamiento intermitente. Revista SdS. 2004;60(61):90-6.

21. Billat V, Bocquet B, Slawinski J, Laffite L. Effects of a prior intermittent run at vVO2MAX on oxygen kinetics during an all-out severe run in humans. J Sports Med Phys Fitness. 2000;40(3):185-94.

22. Rowland T. Entrenamiento del sistema cardiorrespiratorio durante la infancia. Rev Edu Fís. 2015;32(4).

23. Berthoin S, Mantéca F, Gerbeaux M, Lensel-Corbeil. Effect of a 12-week training programme on maximal aerobic speed (MAS) and running time to exhaustion at $100 \%$ of MAS for students aged 14 to 17 years. J Sports Med Phys Fitness. 1995;35:251-6.

24. Reyes T. Efecto de un programa de actividad física sobre el rendimiento aeróbico en jóvenes. Revista Ciencias de la Actividad Física. 2015;16(1):53-61. 